\title{
SINGULAR WEYL-TITCHMARSH-KODAIRA THEORY FOR JACOBI OPERATORS
}

\author{
JONATHAN ECKHARDT AND GERALD TESCHL
}

\begin{abstract}
We develop singular Weyl-Titchmarsh-Kodaira theory for Jacobi operators. In particular, we establish existence of a spectral transformation as well as local Borg-Marchenko and Hochstadt-Liebermann type uniqueness results.
\end{abstract}

Mathematics subject classification (2010): Primary 47B36, 34B20; Secondary 47B39, 34A55. Keywords and phrases: Jacobi operators, inverse spectral theory, discrete spectra.

\section{REFERENCES}

[1] C. Bennewitz, A Paley-Wiener theorem with applications to inverse spectral theory, in Advances in differential equations and mathematical physics (Birmingham, AL, 2002), 21-31, Contemp. Math. 327, Amer. Math. Soc., Providence, RI, 2003.

[2] L. DE BRAnges, Hilbert spaces of entire functions, Prentice-Hall, Inc., Englewood Cliffs, N.J. 1968.

[3] J. ECKHARDT, Inverse uniqueness results for Schrödinger operators using de Branges theory, Complex Anal. Oper. Theory (to appear)

[4] J. ECKHARDT, Direct and inverse spectral theory of singular left-definite Sturm-Liouville operators, J. Differential Equations 253, 2 (2012), 604-634.

[5] J. Eckhardt, F. Gesztesy, R. Nichols, And G. Teschl, Weyl-Titchmarsh theory for SturmLiouville operators with distributional potential coefficients, arXiv:1208.4677.

[6] J. ECKHARDT AND G. TESCHL, Uniqueness results for one-dimensional Schrödinger operators with purely discrete spectra, Trans. Amer. Math. Soc. to appear.

[7] C. Fulton, Titchmarsh-Weyl m-functions for second order Sturm-Liouville problems, Math. Nachr. 281 (2008), 1417-1475.

[8] C. Fulton AND H. LANGer, Sturm-Liouville operators with singularities and generalized Nevanlinna functions, Complex Anal. Oper. Theory 4 (2010), 179-243.

[9] F. Gesztesy, A. Kiselev, AND K. A. MAKAROV, Uniqueness results for matrix-valued Schrödinger, Jacobi, and Dirac-type operators, Math. Nachr. 239-240 (2002), 103-145.

[10] F. GesZTESY AND M. ZinchenKo, On spectral theory for Schrödinger operators with strongly singular potentials, Math. Nachr. 279 (2006), 1041-1082.

[11] I. S. KAC, The existence of spectral functions of generalized second order differential systems with boundary conditions at the singular end, AMS Translations (2) 62 (1967), 204-262.

[12] K. KodAira, The eigenvalue problem for ordinary differential equations of the second order and Heisenberg's theory of S-matrices, Amer. J. Math. 71 (1949), 921-945.

[13] A. Kostenko And G. Teschl, On the singular Weyl-Titchmarsh function of perturbed spherical Schrödinger operators, J. Diff. Eqs. 250 (2011), 3701-3739.

[14] A. KostenKo AND G. TESCHL, Spectral asymptotics for perturbed spherical Schrödinger operators and applications to quantum scattering, arXiv:1205.5049.

[15] A. Kostenko, A. Sakhnovich, And G. Teschl, Inverse eigenvalue problems for perturbed spherical Schrödinger operators, Inverse Problems 26 (2010), 105013, 14pp.

[16] A. Kostenko, A. Sakhnovich, And G. Teschl, Weyl-Titchmarsh theory for Schrödinger operators with strongly singular potentials, Int. Math. Res. Not. 2012 (2012), 1699-1747.

[17] A. Kostenko, A. SAKhnovich, AND G. Teschl, Commutation methods for Schrödinger operators with strongly singular potentials, Math. Nachr. 285 (2012), 392-410. 
[18] P. Kurasov And A. Luger, An operator theoretic interpretation of the generalized TitchmarshWeyl coefficient for a singular Sturm-Liouville problem, Math. Phys. Anal. Geom. Math. 14 (2011), $115-151$.

[19] B. YA. Levin, Lectures on Entire Functions, Transl. Math. Monographs 150, Amer. Math. Soc., Providence, RI, 1996.

[20] M. Rosenblum And J. Rovnyak, Topics in Hardy classes and univalent functions, Birkhäuser Verlag, Basel, 1994.

[21] G. TESCHL, Trace formulas and inverse spectral theory for Jacobi operators, Comm. Math. Phys. 196 (1998), 175-202.

[22] G. TeSCHL, Jacobi Operators and Completely Integrable Nonlinear Lattices, Math. Surv. and Mon. 72, Amer. Math. Soc., Rhode Island, 2000.

[23] R. WEIKARD, A local Borg-Marchenko theorem for difference equations with complex coefficients, in "Partial differential equations and inverse problems", 403-410, Contemp. Math., 362, Amer. Math. Soc., Providence, RI, 2004. 